Proyecciones

Vol. 20, Nº 2, pp. 177-191, August 2001.

Universidad Católica del Norte

Antofagasta - Chile

\title{
SEPARATION PROBLEM FOR STURM-LIOUVILLE EQUATION WITH OPERATOR COEFFICIENT
}

\author{
Z. OER \\ Yildiz Technical University, Turkey
}

\begin{abstract}
Let $H$ be a separable Hilbert Space. Denote by $H_{1}=L_{2}(a, b ; H)$ the set of function defned on the interval $a<x<b(-\infty \leq$ $a<x<b \leq \infty)$ whose values belong to $H$ strongly measurable [12] and satisfying the condition

$$
\int_{a}^{b}\|f(x)\|_{H}^{2} d x<\infty
$$

If the inner product of function $f(x)$ and $g(x)$ belonging to $H_{1}$ is defined by

$$
(f, g)_{1}=\int_{a}^{b}(f(x), g(x))_{H} d x
$$

then $H_{1}$ forms a separable Hilbert space. We study separation problem for the operator formed by $-y^{\prime \prime}+Q(x) y$ SturmLiouville differential expression in $L_{2}(-\infty, \infty ; H)$ space has been proved where $Q(x)$ is an operator which transforms at $H$ in value of x,self-adjoint,lower bounded and its inverse is complete continous.
\end{abstract}




\section{Introduction}

Let us consider

$$
-y^{\prime \prime}+Q(x) y
$$

differential expression in $H_{1}=L_{2}(-\infty, \infty ; H)$. Let us assume that $Q(x)$, satisfies the following conditions as a self-adjoint operator making transformation at $H$ in each value obtained $(-\infty, \infty)$ interval of $x$

1) $Q(x)$ operator family have the same definition set indepent of $\mathrm{x}(-\infty<x<\infty)$ Let us show this set by $\mathcal{D}$.

2) Let $Q(x) \geq I$ and for $\forall f \in \mathcal{D} Q(x) f$ be a strong countinous function in $(-\infty, \infty)$ and $Q^{-1}(x)$ is completely continous in $\mathrm{H}$ for $\forall f \in(-\infty, \infty)$

3) When $|x-y| \leq 2$ let

$$
\left\|(Q(x)-Q(y)) Q^{-1}(y)\right\|<\delta
$$

$\delta>0$ is any number.

Let us form $L_{0}$ operator by (2) expression.Let the definition set $\mathcal{D}\left(L_{0}\right)$ of $L_{0}$ be by the functions $y(x)$ satisfying the following conditions:

1) Let $y(x)$ having compact support in $(-\infty, \infty), Q(x) y(x), y^{\prime \prime}(x)$ be continious.

2) Let $-y^{\prime \prime}(x)+Q(x) y(x) \in L_{2}(-\infty, \infty ; H)$.

We have formed

$$
L_{0} y=-y^{\prime \prime}+Q(x) y \quad, \quad y \in \mathcal{D}\left(L_{0}\right)
$$

Since $H$ which is the definition set of $Q(x)$ is dense almost everywhere and since $Q(x) f$ is continuous at $(-\infty, \infty) \forall f \in \mathcal{D}$ definition set $\mathcal{D}\left(L_{0}\right)$ of $L_{0}$ operator forms a dense linear monifold almost everywhere of the space $L_{2}(-\infty, \infty ; H) . L_{0}$ is a symmetric operator bounded from below in $L_{2}(-\infty, \infty ; H)$ Let us assume that $L$ which is a closure of $L_{0}$ is a self-adjoint operator.

In this work we study the seperability of the operator $L$. According to the definition of seperability when $y(x)$ is any function belonging 
to $\mathcal{D}(L)$ we will show that $y^{\prime \prime}(x)$ and $Q(x) y(x)$ functions also belong to $L_{2}(-\infty, \infty ; H)$ space.

Let us show the resolvent of $L$ operator in reguler $\lambda$ value $(\lambda$ is complex number) by $R_{\lambda}=(L-\lambda I)^{-1}$. According to the definition of Resolvent, $R_{\lambda}$ operator is bounded operator in $H_{1}$ space.

Lemma 1 : [9] If $Q(x) R_{\lambda}$ operator is bounded in $H_{1}$, then $L$ operator is seperable in $H_{1}$.

Proof: Let $y(x)$ be an arbitrary element belonging to $\mathcal{D}(L)$ then $(L-\lambda I) y=f \quad f \in H_{1}$. We can write $y=R_{\lambda} f$ and $Q(x) y=Q(x) R_{\lambda} f$ Since $Q(x) R_{\lambda}$ is a bounded operator, $Q(x) R_{\lambda} f \in H_{1}$ i.e $Q(x) y \in H_{1} \bullet$

Many books, by B. M. Levitan and I. S. Sargsyan [8], E. C. Titcmarch [11], M. Otelbayev [10], and papers by T.C.Fulton and S.A.Pruess [6] belonging to singular Sturm-Liouville problem have been written. English mathematicians W. N. Everitt and M. A. Giertz [3], [4], [5] have proved that they introduced separation definition for operator $L$ consisting of expression (1) being real valued function $Q(x)$ with series papers and studies, and separation theorem of $Q(x)$ for operator $L$ in various conditions. For the separability problem, there are works by M. Bayramoglu and A. Abudov [1], K. Boymatov [2], A. Izmaylov and M. Otelbayev [13], M. Otelbayev [14] and many mathematicians; it has taken big place in the book [9] by the K. Minbayev and M. Otelbayev and given many references in the book.

Localization method for seperability of $L$ operator. This method was firstly used by R.Ismagilov [7], and were developed by M.Otelbayev [10]. We use that developed method.

Let us show the operator $L_{j}$,formed by the following differential expression

$$
-y^{\prime \prime}+Q(x) y \quad, \quad j-1<x<j+1
$$

and

$$
y(j-1)=y(j+1)=0 \quad j=0, \pm 1, \pm 2, \ldots \text { are integers }
$$


boundary conditions in space $L_{2}(j-1, j+1 ; H)$. Each $L_{j}$ operator is a positive defined self-adjoint operator.

Let $w(x)$ be a function satisfying the following properties and differentiable defined in $(-\infty, \infty)$

Let

$$
w(x)= \begin{cases}1 & |x| \leq 1 \\ 0 & |x| \geq 1.5\end{cases}
$$

and $\varphi_{j}(x)=w(x-j)$.

Let us show

$$
\psi_{j}(x)=\left\{\begin{array}{cc}
\frac{1}{2} & |x-j| \leq 1 \\
0 & |x-j|>1
\end{array}\right.
$$

It is seen easily that

$$
\sum_{j=-\infty}^{\infty} \psi_{j}(x)=1
$$

Let $f \in H_{1} \cdot \lambda>0$ let us show

$$
M_{\lambda} f=\sum_{j=-\infty}^{\infty} \varphi_{j}\left(L_{j}+\lambda I\right)^{-1} \psi_{j} f
$$

operator by $M_{\lambda}$.

$$
(L+\lambda I) M_{\lambda} f=\sum_{j=-\infty}^{\infty}(L+\lambda I) \varphi_{j}\left(L_{j}+\lambda I\right)^{-1} \psi_{j} f
$$

Since $L+\lambda I$ and $L_{j}+\lambda I$ operators coincide in the interval $(j-$ $1.5, j+1.5)$ and $\varphi_{j}(x)$ has compact support in the interval $(j-1.5, j+$ $1.5)$, we can write

$$
\begin{aligned}
& (L+\lambda I) M_{\lambda} f= \\
& -\sum_{j=-\infty}^{\infty}\left[\varphi^{\prime \prime}{ }_{j}\left(L_{j}+\lambda I\right)^{-1} \psi_{j} f+2 \varphi_{j}^{\prime} \frac{d}{d x}\left(L_{j}+\lambda I\right)^{-1} \psi_{j} f\right] \\
& +\sum_{j=-\infty}^{\infty} \varphi_{j}\left(L_{j}+\lambda I\right)\left(L_{j}+\lambda I\right)^{-1} \psi_{j} f \\
& =B_{\lambda} f+\sum_{j=-\infty}^{\infty} \varphi_{j} \psi_{j} f
\end{aligned}
$$


where

$$
B_{\lambda} f=\sum_{j=-\infty}^{\infty}\left[\varphi_{j}^{\prime \prime}\left(L_{j}+\lambda I\right)^{-1} \psi_{j} f+2 \varphi_{j}^{\prime} \frac{d}{d x}\left(L_{j}+\lambda I\right)^{-1} \psi_{j} f\right]
$$

If we consider $\varphi_{j} \psi_{j}=\psi_{j}$ and condition (4), the expression (6) can be written as

$$
(L+\lambda I) M_{\lambda} f=\left(I+B_{\lambda}\right) f
$$

If we apply $(L+\lambda I)^{-1}$ operator to both side of (7) we can write

$$
M_{\lambda} f=(L+\lambda I)^{-1}\left(I+B_{\lambda}\right) f
$$

Let $\left(I+B_{\lambda}\right)=g \quad$ or $\quad f=\left(I+B_{\lambda}\right)^{-1} g$. Then (8) equality becomes

$$
M_{\lambda}\left(I+B_{\lambda}\right)^{-1} g=(L+\lambda I)^{-1} g
$$

Let us evalute the norm of $B_{\lambda}$ operator transforming in $H_{1}$. Let $f \in H_{1}$

$$
\begin{aligned}
\left\|B_{\lambda} f\right\|^{2} & =\left\|\sum_{j=-\infty}^{\infty}\left[\varphi_{j}^{\prime \prime}\left(L_{j}+\lambda I\right)^{-1} \psi_{j} f+2 \varphi_{j}^{\prime} \frac{d}{d x}\left(L_{j}+\lambda I\right)^{-1} \psi_{j} f\right]\right\|^{2} \\
& \leq 8 \sum_{j=-\infty}^{\infty}\left\|\varphi_{j}^{\prime \prime}\left(L_{j}+\lambda I\right)^{-1} \psi_{j} f\right\|^{2} \\
& +8 \sum_{j=-\infty}^{\infty}\left\|\varphi_{j}^{\prime}\left(L_{j}+\lambda I\right)^{-1} \psi_{j} f\right\|^{2}
\end{aligned}
$$

In (10) equality it is considered that support of $\varphi_{j}^{\prime}$ and $\varphi_{j+k} \quad(k \geq$ 2) functions are not intersected. Let us evaluate the terms of the first sum on the right side of (10)

$$
\begin{aligned}
\left\|\varphi_{j}^{\prime \prime}\left(L_{j}+\lambda I\right)^{-1} \psi_{j} f\right\|^{2} \leq \frac{c^{2}}{\lambda^{2}}\left\|\psi_{j} f\right\|^{2} & =\frac{c^{2}}{\lambda^{2}} \int_{-\infty}^{\infty}\left\|\psi_{j} f\right\|_{H}^{2} d x \\
& =\frac{c^{2}}{\lambda^{2}} \int_{j-1}^{j+1}\|f(x)\|_{H}^{2} d x
\end{aligned}
$$

Thus,

$$
\left\|\varphi_{j}^{\prime \prime}\left(L_{j}+\lambda I\right)^{-1} \psi_{j} f\right\|^{2} \leq \frac{c^{2}}{\lambda^{2}} \int_{j-1}^{j+1}\|f(x)\|_{H}^{2} d x
$$


Second sum is

$$
\begin{aligned}
\left\|\varphi_{j}^{\prime}\left(L_{j}+\lambda I\right)^{-1} \psi_{j} f\right\|^{2} & \leq c^{2}\left\|\frac{d}{d x}\left(L_{j}+\lambda I\right)^{-1}\right\|^{2}\left\|\psi_{j} f\right\|^{2} \\
& \leq c^{2}\left\|\frac{d}{d x}\left(L_{j}+\lambda I\right)^{-1}\right\|^{2} \int_{j-1}^{j+1}\|f(x)\|^{2} d x
\end{aligned}
$$

Let us prove the following lemma.

Lemma 2: $\left\|\frac{d}{d x}\left(L_{j}+\lambda I\right)^{-1}\right\| \leq c \frac{1}{\sqrt{\lambda}} \quad(\lambda>0)$ inequality holds.

Proof: Let us consider $y(j-1)=y(j+1)=0$ by multiply with $y(x)$ both sides of equation $-y^{\prime \prime}+Q(x) y=f$. Then,

$$
\begin{gathered}
\int_{j-1}^{j+1}\left(-y^{\prime \prime}+Q(x) y, y\right) d x=\int_{j-1}^{j+1}(f, y) d x \\
\int_{j-1}^{j+1}\left[\left\|y^{\prime}\right\|+(Q(x) y, y)\right] d x=\int_{j-1}^{j+1}(f, y) d x
\end{gathered}
$$

If we consider in this inequality that $Q(x)=Q^{*}(x) \geq I$ and use the Schwartz inequality, we obtain

$$
\int_{j-1}^{j+1}\left\|y^{\prime}\right\|^{2} \leq\left(\int_{j-1}^{j+1}\|f(x)\|^{2}\right)^{1 / 2}\left(\int_{j-1}^{j+1}\|y(x)\|^{2}\right)^{1 / 2}
$$

Since

$$
y(x)=\left(L_{j}+\lambda I\right)^{-1} f(x)
$$

then

$$
\|y(x)\|_{L_{2}^{j}}^{2} \leq \frac{1}{\lambda^{2}}\|f\|_{L_{2}^{j}}^{2}=\frac{1}{\lambda^{2}} \int_{j-1}^{j+1}\|f(x)\|^{2} d x
$$

Here $L_{2}^{j}=L_{2}(j-1, j+1)$. If we consider

$$
\begin{gathered}
\int_{j-1}^{j+1}\left\|y^{\prime}\right\|^{2} d x \leq \frac{1}{\lambda} \int_{j-1}^{j+1}\|f(x)\|^{2} d x \\
y^{\prime}=\frac{d}{d x}\left(L_{j}+\lambda I\right)^{-1} f \\
\left\|\frac{d}{d x}\left(L_{j}+\lambda I\right)^{-1} f\right\|_{L_{2}^{j}} \leq \frac{1}{\sqrt{\lambda}}\|f\|_{L_{2}^{j}}
\end{gathered}
$$


is obtain from the last inequality. Since $f$ is the arbitary element of $L_{2}^{j}$ we find

$$
\left\|\frac{d}{d x}\left(L_{j}+\lambda I\right)^{-1}\right\|<\frac{1}{\sqrt{\lambda}}
$$

Thus, the terms belonging to the second sum in (10) are

$$
\left\|\varphi_{j}^{\prime} \frac{d}{d x}\left(L_{j}+\lambda I\right)^{-1} \psi_{j} f\right\|^{2} \leq \frac{c}{\lambda} \int_{j-1}^{j+1}\|f(x)\|^{2} d x \quad \lambda \geq 1
$$

If we consider $(10),(11),(12)$ inequalities we find

$$
\begin{aligned}
\left\|B_{\lambda} f\right\|^{2} & \leq \frac{c^{2}}{\lambda^{2}} \sum_{j=-\infty}^{\infty} \int_{j-1}^{j+1}\|f(x)\|^{2} d x+\frac{c}{\lambda} \sum_{j=-\infty}^{\infty} \int_{j-1}^{j+1}\|f(x)\|^{2} d x \\
& \leq \frac{c_{1}}{\lambda} \sum_{j=-\infty}^{\infty} \int_{j-1}^{j+1}\|f(x)\|^{2} d x \\
& =\frac{c_{1}}{\lambda} \sum_{j=-\infty}^{\infty}\left(\int_{j-1}^{j}\|f(x)\|^{2} d x+\int_{j}^{j+1}\|f(x)\|^{2} d x\right) \\
& =\frac{c_{1}}{\lambda} \sum_{j=-\infty}^{\infty} \int_{j-1}^{j}\|f(x)\|^{2} d x+\frac{c_{1}}{\lambda} \sum_{j=-\infty}^{\infty} \int_{j}^{j+1}\|f(x)\|^{2} d x \\
& =\frac{2 c_{1}}{\lambda} \int_{-\infty}^{\infty}\|f(x)\|^{2} d x=\frac{2 c_{1}}{\lambda}\|f\|^{2}
\end{aligned}
$$

Thus,

$$
\left\|B_{\lambda}\right\| \leq 2 \frac{c_{1}}{\lambda}
$$

where in big pozitive values of $\lambda$, it is found that $\left\|B_{\lambda}\right\|$ is as small as desired.Therefore, we can write

$$
M_{\lambda}=(L+\lambda I)^{-1}\left(I+B_{\lambda}\right)
$$

formula as

$$
(L+\lambda I)^{-1}=M_{\lambda}\left(I+B_{\lambda}\right)^{-1}
$$

Lemma 3: If $Q(x) M_{\lambda}$ is bounded, then $L$ operator is separable.

Proof: According to Lemma 1, if $Q(x)(L+\lambda I)^{-1}$ is bounded, then $L$ is separable. By the equation

$$
(L+\lambda I)^{-1}=M_{\lambda}\left(I+B_{\lambda}\right)^{-1}
$$


the proof of Lemma 3 is obtained.

Lemma 4: If $\sup _{j}\left\|Q(x)\left(L_{j}+\lambda I\right)^{-1}\right\|<\infty$, then $Q(x) M_{\lambda}$ operator is bounded.

\section{Proof:}

$$
\begin{aligned}
\left\|Q(x) M_{\lambda} f\right\|^{2} & =\left\|\sum_{j=-\infty}^{\infty} \varphi_{j}(x)(L+\lambda I)^{-1} \psi_{j} f\right\|^{2} \\
& \leq 4 \sum_{j=-\infty}^{\infty}\left\|\varphi_{j} Q(x)(L+\lambda I)^{-1} \psi_{j} f\right\|^{2} \\
& \leq \sum_{j=-\infty}^{\infty}\left\|\varphi_{j} Q(x)(L+\lambda I)^{-1}\right\|^{2}\left\|\psi_{j} f\right\|^{2} \\
& \leq \sup _{j}\left(\left\|\varphi_{j} Q(x)(L+\lambda I)^{-1}\right\|^{2}\right) \sum_{j=-\infty}^{\infty}\left\|\psi_{j} f\right\|^{2} \\
& =2 A\|f\|_{H_{1}}^{2}
\end{aligned}
$$

Thus, $\left\|Q(x) M_{\lambda} f\right\|^{2} \leq 2 A\|f\|^{2}$. From this we find

$$
\left\|Q(x) M_{\lambda}\right\| \leq \sqrt{2 A}
$$

and Lemma is proved. Let us show that $\left\|Q(x)\left(L_{j}+\lambda I\right)^{-1}\right\|$ is finite. When $|x-y| \leq 2$ let us assume that

$$
\left\|(Q(x)-Q(y)) Q^{-1}(y)\right\| \leq \delta
$$

$(\delta=$ const. $>0)$ Let us consider the following boundary value problem

$$
\begin{gathered}
-y^{\prime \prime}+Q(x) y=f \\
y(j-1)=y(j+1)=0
\end{gathered}
$$

Let us write this problem as

$$
\begin{gathered}
-y^{\prime \prime}+(-Q(j)+Q(x)) y+Q(j) y=f \\
y(j-1)=y(j+1)=0
\end{gathered}
$$


Let

$$
-y^{\prime \prime}+Q(j) y=v
$$

and

$$
A_{j} y=-y^{\prime \prime}+Q(j) y
$$

Then $v=A_{j} y$. If we consider these, we can write (14) as

$$
\begin{aligned}
f=L_{j} y & =A_{j} y+(Q(x)-Q(j)) y(15) \\
v+(Q(x)-Q(j)) A_{j}^{-1} v & =\left(I+(Q(x)-Q(j)) A_{j}^{-1}\right) v(1.2)
\end{aligned}
$$

Let us evaluate the norm of operator $(Q(x)-Q(j)) A_{j}^{-1}$. For this let us write the expression as

$$
(Q(x)-Q(j)) A_{j}^{-1}=(Q(x)-Q(j)) Q(j)^{-1}\left(Q(j) A_{j}^{-1}\right)
$$

If we use the condition (13) we can write

$$
\left\|(Q(x)-Q(j)) A_{j}^{-1}\right\| \leq \delta\left\|Q(j) A_{j}^{-1}\right\|
$$

Now let us prove the following Lemma. 1.

Lemma 5: The set of $Q(j) A_{j}^{-1}$ operator is smooth bounded with

$$
\left\|Q(j) A_{j}^{-1}\right\| \leq 1
$$

Proof: We will prove the Lemma by using opening formula according to eigenvector of $Q(j)$ operator.

Let us find $A_{j}^{-1}$ operator in order to show that $\left\|Q(j) A_{j}^{-1}\right\| \leq 1$

Let us consider

$$
\begin{gathered}
A_{j} y=-y^{\prime \prime}+Q(j) y=f(x) f(x) \in L_{2}(j-1, j+1 ; H) \\
y(j-1)=0 \quad y(j+1)=0
\end{gathered}
$$


where $Q^{-1}(j)$ complete continous and therefore its spectrum is pure-disjoint.

Let eigenvalues of $Q(j)$ be

$$
\alpha_{1}(j) \leq \alpha_{2}(j) \leq \ldots
$$

and the corresponding eigenvectors to these eigenvalues be

$$
g_{1}(j), g_{2}(j), \ldots
$$

These form a base in $H$.

$$
\begin{array}{ll} 
& (x) \\
& = \\
\sum_{k=1}^{\infty} h_{k}(x) g_{k}(j) & h_{k}(x)=\left(f(x), g_{k}(j)\right) \\
& y_{j}(x) \\
& = \\
\sum_{k=1}^{\infty} y_{k, j}(x) g_{k}(j) & y_{k, j}(x)=\left(y(x), g_{k}(j)\right)
\end{array}
$$

If we put these equations in (17) we find

$$
\begin{gathered}
(20)-\sum_{k=1}^{\infty} y_{k}^{\prime \prime}(x) g_{k}(j)+\sum_{k=1}^{\infty} \alpha_{k}(j) y_{k}(x) g_{k}(j)=\sum_{k=1}^{\infty} h_{k}(x) g_{k}(j) \\
-y_{k}^{\prime \prime}(x)+\alpha_{k}(j) y_{k}=h_{k}(x) \quad k=1,2, \ldots \\
\left.\sum_{k=1}^{\infty} y_{k}(x) g_{k}(j)\right|_{x=j-1}=0 \quad,\left.\quad \sum_{k=1}^{\infty} y_{k}(x) g_{k}(j)\right|_{x=j+1}=0 \\
\sum_{k=1}^{\infty} y_{k}(j-1) g_{k}(j)=0 \quad, \quad \sum_{k=1}^{\infty} y_{k}(j+1) g_{k}(j)=0
\end{gathered}
$$

Thus, (17),(18) problems are transformed

$$
\begin{gathered}
-y_{k}^{\prime \prime}(x)+\alpha_{k}(j) y_{k}=h_{k}(x) \\
y_{k}(j-1)=0 \quad, \quad y_{k}(j+1)=0
\end{gathered}
$$


The eigenvalues of these problems are

$$
\lambda=\frac{k^{2} \pi^{2}}{4}
$$

The corresponding normalized eigenvectors are the following:

if $j$ is odd;

$$
y=\sin \frac{k \pi}{2} x
$$

if $j$ even and $k$ is odd;

$$
y_{k, j}=\cos \frac{k \pi}{2} x
$$

if $k$ is even;

$$
\begin{gathered}
y_{k, j}=\sin \frac{k \pi}{2} x \\
y_{k, j}=\sum_{m=0}^{\infty} \frac{1}{\lambda_{m}+\alpha_{k}(j)}\left(h_{k}, \varphi_{m, j}\right) \varphi_{m, j} \\
\left(h, \varphi_{m}\right)=\int_{j-1}^{j+1} h(x) \varphi_{m}(x) d x
\end{gathered}
$$

Let us consider (23) in (19)

$$
\begin{gathered}
y_{j}(x)=\sum_{k=1}^{\infty}\left(\sum_{m=0}^{\infty} \frac{1}{\lambda_{m}+\alpha_{k}(j)}\left(h_{k}, \varphi_{m, j}\right) \varphi_{m, j}\right) g_{k}(j) \\
Q(j) A_{j}^{-1} f=Q(j) y_{j}(x) \\
=Q(j) \sum_{k=1}^{\infty}\left(\sum_{m=0}^{\infty} \frac{1}{\lambda_{m}+\alpha_{k}(j)}\left(h_{k}, \varphi_{m, j}\right) \varphi_{m, j}\right) g_{k}(j) \\
=\sum_{k=1}^{\infty}\left(\sum_{m=0}^{\infty} \frac{1}{\lambda_{m}+\alpha_{k}(j)}\left(h_{k}, \varphi_{m, j}\right) \varphi_{m, j}\right) Q(j) g_{k}(j) \\
=\sum_{k=1}^{\infty}\left[\sum_{m=0}^{\infty} \frac{\alpha_{k}(j)}{\lambda_{m}+\alpha_{k}(j)}\left(h_{k}, \varphi_{m, j}\right) \varphi_{m, j}\right] g_{k}(j)
\end{gathered}
$$


Thus,

$$
Q(j) f=A_{j}^{-1} f=\sum_{k=1}^{\infty}\left[\sum_{m=0}^{\infty} \frac{\alpha_{k}(j)}{\lambda_{m}+\alpha_{k}(j)}\left(h_{k}, \varphi_{m, j}\right) \varphi_{m, j}\right] g_{k}(j)
$$

Since $\left\{\varphi_{m, j}(x)\right\}_{m=0}^{\infty}$ functions form a complete ortonormal system in $L_{2}(j-1, j+1)$, and the element $\left\{g_{k}(j)\right\}_{j=1}^{\infty}$ form a complete ortonormal system in $H \quad\left\{\varphi_{m, j}(x) g_{k}(j)\right\}_{m=0, k=1}^{\infty}$ forms a complete ortonormal system in Hilbert space.

Since the system $\left\{\varphi_{m, j}(x) g_{k}(j)\right\}_{m=0, k=1}^{\infty}$ forms a complete ortonormal system in $L_{2, j}=L_{2}(j-1, j+1 ; H)$ if we use Parseval equation we can write from $(24)$

$$
\left\|Q(j) A_{j}^{-1} f\right\|^{2}=\sum_{k=1}^{\infty} \sum_{m=0}^{\infty}\left(\frac{\alpha_{k}(j)}{\lambda_{m}+\alpha_{k}(j)}\right)^{2}\left|\left(h_{k}, \varphi_{m, j}\right)\right|^{2}
$$

Since $\alpha_{k}(j) \geq 1, \frac{\alpha_{k}(j)}{\lambda_{m}+\alpha_{k}(j)}<1$ and

$$
\left\|Q(j) A_{j}^{-1} f\right\|^{2} \leq \sum_{k=1}^{\infty} \sum_{m=0}^{\infty}\left|\left(h_{k}, \varphi_{m, j}\right)\right|^{2}=\|f\|^{2}
$$

or

$$
\left\|Q(j) A_{j}^{-1} f\right\| \leq\|f\|
$$

that is $\left\|Q(j) A_{j}^{-1}\right\| \leq 1$. Thus, we have proved $Q(j) A_{j}^{-1}$ operators are straight bounded.

According to Lemma, (16) becomes

$$
\left\|(Q(x)-Q(j)) A_{j}^{-1}\right\| \leq \delta
$$

Let us assume that $\delta<1$. Then from (15) we find

$$
v=\left(I+(Q(x)-Q(j)) A_{j}^{-1}\right)^{-1} f
$$

Let

$$
T_{j}=\left(I+(Q(x)-Q(j)) A_{j}^{-1}\right)^{-1}
$$

The last expression becomes

$$
v=T_{j} f
$$


and we obtain

$$
y(x)=A_{j}^{-1} T_{j} f
$$

Thus

$$
\begin{array}{rlcl}
Q(x) y(x) \| & = & \left\|\left(Q(x) Q^{-1}(j)\right)\left(Q(j) A_{j}^{-1} T_{j} f\right)\right\| \\
& \leq & \left\|Q(x) Q^{-1}(j)\right\| c\left\|T_{j} f\right\| \\
& \leq & \left\|Q(x) Q^{-1}(j)\right\| c_{1}\|f\|_{L_{2, j}}
\end{array}
$$

If we consider here condition

$$
\left\|(Q(x)-Q(j)) Q^{-1}(j)\right\| \leq \delta
$$

then we find

$$
\left\|Q(x) Q^{-1}(j)-I\right\| \leq \delta
$$

Therefore, inequality (26) becomes

$$
\|Q(x) y(x)\| \leq c_{2}\|f\|_{L_{2, j}}
$$

Here, if we consider $y(x)=L_{j}^{-1} f$, the last inequality takes the form of

$$
\left\|Q(x) L_{j}^{-1} f\right\| \leq c_{2}\|f\|_{L_{2, j}}
$$

If we consider, then we find

$$
\left\|Q(x)\left(L_{j}+\lambda I\right)^{-1} f\right\| \leq c_{2}\|f\|_{L_{2, j}}
$$

or

$$
\left\|Q(x)\left(L_{j}+\lambda I\right)^{-1}\right\| \leq c
$$

Thus, we have proved the theorem of separability.

Theorem: When the conditions 1) - 3) are satisfied, $L$ operator is separable in $H_{1}$.

\section{Acknowledgement}

I express deep gratitude to M.Bayramoglu and M.Otelbayev for their attenition to the work 


\section{References}

[1] Bayramoglu, M., Abudov, A. A., About Sturm-Liouville Operator With Operator Coefficient is Essential Self-Adjoint, Sbornik, Spectral Theory of Operators, Bak, "Elm", pp. 12-20, (1982).

[2] Boymatov, K. Ch., Separable Theorems For Sturm-Liouville Operator, Matem. Zametki, Vol.14, No:3, pp. 349-359, (1973).

[3] Everitt, W. N., Giertz, M. A., Dirichlet Type Result For Ordinary Differential Operators, Math. Ann., Vol. 203, No 2, pp. 119-128, (1973).

[4] Everitt, W.N., Giertz, M.A., An Example Conserning The Separation Property For Differential Operators, Proc.Roy.Soc. Edinburg A, Vol.71, pp. 159-165, (1973).

[5] Everitt, W.N., Giertz, M.A., Inequalities And Separation For Schrdinger Type Operators in $L_{2}\left(R_{n}\right)$, Proc.Roy.Soc. Edinburg A, Vol.79, pp. 257-265, (1977).

[6] Fulton, T. C., Pruess, S. A., Eigenvalue And Eigenfunction Asymtotics For Regular Sturm-Liouville Problems, J. Math. Anal. Appl., 188, pp. 297-340, (1994).

[7] Ismagilov, R. S., Self-adjointless of the Sturm-Liouville Operator UMN, 18, No 5, pp. 161-166, (1963).

[8] Levitan, B. M. and Sargsyan, I. S., Strum-Liouville And Dirac Operators, Kluzer, Dordrechz, (1991).

[9] Minbayev, K. T., Otelbayev, M. O., The Weighted of Functional Spaces and Spectrum of the Differential Operators, Moskova "Nauka", (1988) (R).

[10] Otelbayev, M. O., Behaviour of Spectrum of the Sturm-Liouville operator, Alma Ata. Bilim (1990). 
[11] Titchmarsh, E. C., Eigenfunctions Expansions Associated With Second Order Differential Equations, 2nd ed., Vol.I, Oxford Univ.Press, London, (1962).

[12] Yosida, K., Functional Analysis, Berlin-Gttingen-Heidelberg: Springer-Verlag, (1980).

[13] Izmaylov, A. L., Otelbayev, M. O., Weighted Integrability of Solutions of Differential Equation Given in Bounded Area, Izv. An Kaz. SSR., Ser. Fiz. Mat, No 1, pp. 36-40, (1977).

[14] Otelbayev, M. O., Weighted Integrability of Solutions Of SturmLiouville Equation, Matem. Zametki, Vol. 16, No 6, pp. 969-980, (1974).

Received : July 2000.

\section{Z. OER}

Department of Mathematics

Faculty of Art and Science

Yildiz Technical University

34210, Davutpasa

Istanbul

Turkey

E-MAIL : zoer@yildiz.edu.tr 